\title{
MELD-XI Score Predict No-Reflow Phenomenon and Short-Term Mortality in Patient With ST-Segment Elevation Myocardial Infarction Undergoing Primary Percutaneous Coronary Intervention
}

\author{
Xin-Tao Zhang \\ Union Hospital, Fujian Medical University \\ Zhao-Rong Lin \\ Union Hospital, Fujian Medical University \\ Lin Zhang \\ Union Hospital, Fujian Medical University \\ Zi-Wen Zhao \\ Union Hospital, Fujian Medical University \\ Liang-Long Chen ( $\sim$ lianglongchenxh@126.com ) \\ Union Hospital, Fujian Medical University
}

\section{Research Article}

Keywords: No-reflow phenomenon, MELD-XI, Short-term outcome, ST-segment elevation myocardial infarction, liver and renal dysfunction, Percutaneous coronary intervention

Posted Date: October 28th, 2021

DOI: https://doi.org/10.21203/rs.3.rs-1009646/v1

License: (c) (1) This work is licensed under a Creative Commons Attribution 4.0 International License.

Read Full License

Version of Record: A version of this preprint was published at BMC Cardiovascular Disorders on March 18th, 2022. See the published version at https://doi.org/10.1186/s12872-022-02556-2. 


\section{Abstract \\ Introduction}

: No-reflow phenomenon (NRP) is one of high-incidence complication during percutaneous coronary intervention $(\mathrm{PCl})$. In the current study, we examined the relationship between MELD-XI score and NRP in detail. Moreover, we further discuss whether MELD-XI score could consider as an accurate risk assessment score of patients with STEMI candidate for PCI.

\section{Methods}

This retrospective study involving 693 Patients with acute STEMI underwent an emergency PCI were consecutive recruited. All patients divided into normal reflow or no-reflow groups based in result of post interventional thrombolysisin myocardial in farction(TIMI) flow rate. Univariate and multivariate logistic regression and Cox regression analysis were conducted to identify the independent predictors of determine the independent predictive factor in both group. Receiver operator characteristic (ROC) curve and Kaplan-Meier (K-M) have been applied to estimate the predictive values of MELD-XI score.

\section{Results}

MELD-XI score is a independently indicator of NRP(odds ratio(OR):1.247, 95\%Cl:1.144-1.360, P<0.001). A multivariate Cox regression analysis also revealed that MELD-XI score is an independent prognostic factor for 30-days all-cause mortality(hazard ratio(HR):1.155, 95\%Cl:1.077-1.239, $\mathrm{P}<0.001$ ). Moreover, the cutoff value of MELD-XI score to predict NRP was 9.47 by ROC curve, area under ROC curve(AUC):0.739, $\mathrm{P}<0.001$. Kaplan-Meier curves for 30-days all cause mortality revealed lower survival rate in MELD-XI $\llbracket 9.78$ group $(P \otimes 0.001)$.

\section{Conclusion}

The MELD-XI score predict NRP and 30-days prognosis accurately in STEMI patients whom undergoing $\mathrm{PPCl}$. It could as an inexpensive and readily available tool for risk stratification.

\section{Introduction}

Primary percutaneous coronary intervention(PPCl) could greatly reduce mortality and disability rate of patients with acute ST-segment elevation myocardial infarction(STEMI). However, the NRP still occur frequently in the $\mathrm{PCl}$ treatment of STEMI patients. According to previous research, NRP is an important predictor of poor short-term and long-term adverse outcome for patients underwent PCI[1]. Given the importance of NRP, we eager to establish a fast and effective risk stratification method for acute STEMI patients candidated to PPCl. 
The MELD-XI (the model for end-stage liver disease excluding international normalized ratio) score was originally used to evaluate derangements of liver and renal function. Furthermore, MELD-XI has been found to be associated with poor prognosis of heart failure in recent plethora research[2]. Renal function status is independently correlated with the mortality rate of elderly STEMI patients underwent PCI[3]. Bilirubin levels are not only independently related to no-reflow during hospitalization, but also in-hospital major adverse clinical events(MACEs)[4]. Elevated serum creatinine at admission is also one of the risk factors for NRP[5, 6].

We aim to assess the MELD-XI score calculated on admission whether predict NRP and 30-days prognosis accurately in patients with STEMI undergoing PPCI.

\section{Methods}

\subsection{Study population}

In this study, we retrospectively enrolled 693 consecutive patients presenting with STEMI admitted to our emergency cardiovascular department appropriate for PPCI January 2018 and July 2021. Infarct-related artery blood flow were evaluated as per the thrombolysis in the myocardial infarction (TIMI) flow grade. According to post-PCI TIMI flow grade, the study population was subdivided into no-reflow group(TIMI flow grade of $\llbracket 2$ ) and normal reflow group(TIMI flow grade of $\geq 2$ ).

The definition of the TIMI flow grades is classically as follows: Grade 0 refer to there is no antegrade blood flow through vessel under the angiography after the vascular completely occluded. Grade 1, The contrast agent not be completely blocked, a small amount of the contrast material flow pass through the occlusion site, but the distal coronary artery cannot be filled. Grade 2 , the contrast agent could fill the distal coronary vessels, but the blood flowis slower than the grade 3,and grade 3 , same as normal coronary blood flow.

According to the relevant guideline definitions, the diagnostic criteria for acute STEMI in this study are as follows:(1)The typical symptoms of angina pectoris symptoms located behind the sternum not completely relieved even if rest or(and) take nitroglycerin drugs for more than 30 minutes; (2)Patients with a new st-segment elevation in at least two consecutive leads or left bundle branch block on the electrocardiogram (ECG); (3)The levels of myocardial injury markers, including the typical increase in troponin T or troponin I levels within 12 hour after onset of symptoms[7].

The exclusion criteria were: (1)patients who had previous acute thrombolytic therapy before coming to emergency department; (2) patients who reject percutaneous stent implantation; (3) patients with endstage liver cirrhosis or severe kidney disease undergoing dialysis; (4)presence of infectious or chronic inflammatory-autoimmune disease or malignancy.

\subsection{Coronary angiography and procedure}


All patients performed via the radial artery were prescribed load-dose drugs of aspirin (300 $\mathrm{mg})$, ticagrelor $(180 \mathrm{mg})$ or clopidogrel $(600 \mathrm{mg})$ before PPCl. Coronary angiography was performed using standard techniques through radial approach for all patients. The various parameters and methods of the procedure operation are determined by the individual operators

The angiogram results and the post-PCI thrombolysis in myocardial infarction (TIMI) flow grade were analyzed independently and in a double-blind manner by two interventional cardiologists. The no-reflow phenomenon defined as a coronary TIMI grade flow $₫ 2$ after stent implantation[9]. A residual stenosis under $20 \%$ and TIMI flow grade $\geq 2$ for the treated coronary vessel was considered as successful PPCI.

\subsection{Measurement, Data collection and endpoint}

Fast blood samples were obtained from all subjects prior to coronary angiography. Serum serum high sensitivity C-reactive protein (hs-CRP), total cholesterol(TC), high density lipoprotein cholesterol (HDL-C), low density lipoprotein cholesterol (LDL-C) and triglycerides(TG), uric acid(UA), serum albumin(ALB), Creatine Kinase-MB (CK-MB), N terminal pro B type natriuretic peptide(Nt-proBNP), serum creatine( $\mathrm{sCr}$ ), serum total bilirubin(TB)levels were measured. Left ventricular ejection fraction (LVEF) was obtained from the clinical echocardiographic report before coronary angiogram.

Two researchers collected relevant demographic and clinical characteristics of all patients from hospital files and electronic medical records and check for errors. Then, another researcher conducted to analyze all data. The calculated formula of MELD-XI score as shown: $5.11 \times(\ln$ total bilirubin, $\mathrm{mg} / \mathrm{dL})+11.76 \times(\mathrm{In}$ creatinine, $\mathrm{mg} / \mathrm{dL})+9.44[10]$.

The primary endpoints were 30-days all-cause mortality. Discharged patient were reviewed via telephonic interview. The research protocol was approved by the Ethics Committee of the Union Hospital affiliated to Fujian Medical University. All procedure in accordance with the principles outlined in the Helsinki Declaration.

\subsection{Statistical analysis}

Kolmogorov-Smirnov test was used to evaluate whether the distribution of continuous variables was normal. Continuous data with a normal distribution are expressed as mean \pm standard deviation(SD), The median and interquartile range (IQR) were used to describe for continuous variables with a skewed distribution. Continuous variables were compared using an unpaired Student's t-test or Manne-Whitney Utest. Categorical variables were presented as frequency and compared using the chi-square test or Fisher exact test.

The potential predictors of NRP and 30-days mortality were examined by univariate and multivariate analyses. The area under ROC curve (AUC) were used for evaluating the predictive value of MELD-XI scores. Two-tailed tests were applied in all statistical tests and a $\mathrm{P}<0.05$ was considered statistically significant. The independent predictors of the primary endpoint was analyzed through multivariate Cox proportional-hazards regression model. The Kaplan-Meiercurves were used to analyze the 30-day 
survival, while statistical differences between the two groups were assessed using the log rank test. SPSS 25.0 software (SPSS Inc, IBM Corporation, Armonk, New York, USA) system was used to perform statistical analysis of all data collected.

\section{Result}

The study population's general clinical characteristics of demographic, clinical, laboratory, procedural characteristics were listed in Table 1. The overall study population segregated into two groups: the normal-reflow group $(n=547,457$ men, mean age $62.34 \pm 12.46$ years $)$ and the no-reflow group $(n=146,117$ men, mean age $63.95 \pm 12.38$ years). The incidence of NRP among all the STEMI patients was $21.0 \%$. Regarding demographical and Laboratory parameters, including Heart failure, Peripheral vascular history, Hypertension, Diabetes mellitus, SBP, HDL-C, BUN, sCr, hs-CRP, TB were meaningful differences were observed in Table 1. In addition, the MELD-XI scores in the normal reflow group were significantly lower than in the no-reflow group. However, we did not found meaningful differences between the normal reflow and no-reflow groups with gender, age, Hyperlipidaemia, smoking, LVEF, anterior myocardial infarction, time to hospital $>4 \mathrm{~h}$, multiple stenosis vessels regard to at baseline. 
Table 1

Baseline Characteristics of the study participants

\begin{tabular}{|c|c|c|c|}
\hline Variable & normal(n=547) & no-reflow(n=146) & $\begin{array}{l}P \\
\text { value }\end{array}$ \\
\hline \multicolumn{4}{|l|}{$\begin{array}{l}\text { Clinic and Demographic } \\
\text { characteristics }\end{array}$} \\
\hline Age(years) & $62.34 \pm 12.46$ & $63.95 \pm 12.38$ & 0.181 \\
\hline Male, n(\%) & $457(83.5 \%)$ & $117(80.1 \%)$ & 0.326 \\
\hline Smoking, n(\%) & $369(67.5 \%)$ & $86(58.9 \%)$ & 0.053 \\
\hline Heart failure(Killip II-IV,class),n(\%) & $136(24.9 \%)$ & $54(37.0 \%)$ & 0.004 \\
\hline Hypertension,n(\%) & $306(55.9 \%)$ & $103(70.5 \%)$ & 0.001 \\
\hline Diabetes mellitus,n(\%) & $168(30.7 \%)$ & $73(50.0 \%)$ & $<0.001$ \\
\hline Stroke or TIA,n(\%) & $53(9.7 \%)$ & $15(10.3 \%)$ & 0.876 \\
\hline Family history,n(\%) & $22(4.0 \%)$ & $6(4.1 \%)$ & 0.962 \\
\hline Peripheral vascular history,n(\%) & $11(2.0 \%)$ & $14(9.6 \%)$ & $<0.001$ \\
\hline Atrial fibrillation,n(\%) & $34(6.2 \%)$ & $9(6.2 \%)$ & 0.996 \\
\hline Hyperlipidaemia,n(\%) & $329(60.1 \%)$ & 77(52.7\%) & 0.106 \\
\hline Heart rate $(\mathrm{bpm})$ & $78.00 \pm 15.11$ & $78.46 \pm 15.70$ & 0.699 \\
\hline SBP, mmHg & $125.76 \pm 22.37$ & $130.47 \pm 24.82$ & 0.042 \\
\hline $\mathrm{DBP}, \mathrm{mmHg}$ & $78.04 \pm 13.72$ & $79.86 \pm 16.03$ & 0.202 \\
\hline LVEF(\%) & $50.66 \pm 9.56$ & $49.71 \pm 9.51$ & 0.327 \\
\hline Anterior myocardial infarction, n (\%) & $310(56.7 \%)$ & $81(55.5 \%)$ & 0.796 \\
\hline Time to hospital $>4 \mathrm{~h}$ & $382(69.8 \%)$ & $100(68.5 \%)$ & 0.754 \\
\hline \multicolumn{4}{|l|}{ Laboratory parameters } \\
\hline $\mathrm{TC}(\mathrm{mmol} / \mathrm{L})$ & $4.55 \pm 1.07$ & $4.63 \pm 1.06$ & 0.557 \\
\hline $\mathrm{TG}(\mathrm{mmol} / \mathrm{L})$ & $1.44(1.05-2.08)$ & $1.39(1.02-2.05)$ & 0.056 \\
\hline LDL-C (mmol/L) & $3.03(2.41-3.69)$ & $3.08(2.51-3.72)$ & 0.623 \\
\hline $\mathrm{HDL}-\mathrm{C}(\mathrm{mmol} / \mathrm{L})$ & $0.96(0.82-1.15)$ & $1.05(0.90-1.24)$ & 0.001 \\
\hline
\end{tabular}

SBP: Systolic blood pressure; DBP: diastolic blood pressure; LVEF: Left ventricular ejection fraction; TC: Total cholesterol; TG: Triglycerides; LDL-C: Low density lipoprotein cholesterol; HDL-C: High density lipoprotein cholesterol; BUN: blood urea nitrogen; SCr: serum creatine; hs-CRP: high sensitivity C-reactive protein; CK-MB: Creatine Kinase-MB; UA: uric acid; HCY: homocysteine; TB: Total bilirubin; Nt-proBNP: N-terminal pro-brain natriuretic peptide; LMCA: Left main coronary artery. 


\begin{tabular}{|c|c|c|c|}
\hline Variable & normal $(n=547)$ & no-reflow(n=146) & $\begin{array}{l}P \\
\text { value }\end{array}$ \\
\hline $\mathrm{BUN}(\mathrm{mmol} / \mathrm{L})$ & $4.90(4.00-6.30)$ & $5.75(4.43-7.48)$ & $<0.001$ \\
\hline $\mathrm{sCr}(\mathrm{mg} / \mathrm{dL})$ & $0.83(0.74-0.97)$ & $0.97(0.81-1.19)$ & $<0.001$ \\
\hline hs-CRP (mg/L) & 8.13(3.19-22.60) & $12.10(4.57-32.53)$ & 0.017 \\
\hline CK-MB(U/L) & $88.85(28.53-211.38)$ & 82.85(30.35-201.325) & 0.789 \\
\hline $\mathrm{UA}(\mu \mathrm{mol} / \mathrm{L})$ & $367.19 \pm 108.31$ & $374.88 \pm 111.34$ & 0.465 \\
\hline $\mathrm{HCY}(\mu \mathrm{mol} / \mathrm{L})$ & $9.19(7.37-11.24)$ & $9.89(7.72-12.09)$ & 0.059 \\
\hline $\mathrm{TB}(\mathrm{mg} / \mathrm{dL})$ & $0.72(0.54-0.93)$ & $0.91(0.59-1.19)$ & $<0.001$ \\
\hline Nt-proBNP(pg/ml) & $\begin{array}{l}629.50(181.75- \\
1542.75)\end{array}$ & $\begin{array}{l}807.50(181.25- \\
2104.75)\end{array}$ & 0.056 \\
\hline MELD-XI score & $9.44(9.44-10.29)$ & 10.67(9.65-12.59) & $<0.001$ \\
\hline \multicolumn{4}{|l|}{ Preprocedural characteristics } \\
\hline Multiple stenosis vessels,n(\%) & $345(63.1 \%)$ & $96(65.8 \%)$ & 0.549 \\
\hline LMCA,n(\%) & $36(6.6 \%)$ & $7(4.8 \%)$ & 0.563 \\
\hline \multicolumn{4}{|c|}{$\begin{array}{l}\text { SBP: Systolic blood pressure; DBP: diastolic blood pressure; LVEF: Left ventricular ejection fraction; } \\
\text { TC: Total cholesterol; TG: Triglycerides; LDL-C: Low density lipoprotein cholesterol; HDL-C: High } \\
\text { density lipoprotein cholesterol; BUN: blood urea nitrogen; sCr: serum creatine; hs-CRP: high sensitivity } \\
\text { C-reactive protein; CK-MB: Creatine Kinase-MB; UA: uric acid; HCY: homocysteine; TB: Total bilirubin; } \\
\text { Nt-proBNP: N-terminal pro-brain natriuretic peptide; LMCA: Left main coronary artery. }\end{array}$} \\
\hline
\end{tabular}

Table 2 present the independent predictors of no-reflow. Univariate and Multivariate logistic regression analysis revealed that revealed that Diabetes mellitus(OR:2.173, 95\%Cl:1.423-3.227, $\mathrm{P}<0.001)$, Peripheral vascular history(OR:3.448, 95\%Cl:1.412-8.421, $\mathrm{P}=0.007)$, MELD-XI(OR:1.247, 95\%Cl:1.144-1.360, $\mathrm{P}<0.001)$, Total bilirubin(OR:1.046, 95\%Cl:1.023-1.070, $\mathrm{P}<0.001)$, sCr (OR:1.014, 95\%Cl:1.007-1.021, $\mathrm{P}<0.001$ ) were significant and independent predictors of NRP in our study. ROC curve analyses showed that MELD-XI score had the optimal cut-off value of predicting NRP was 9.47 , with $81.90 \%$ sensitivity and $66.67 \%$ specificity (AUC $=0.739,95 \% \mathrm{Cl} 0.696-0.783, \mathrm{P}<0.001$, Figure $1 \mathrm{a})$. Cox regression multivariate analyses shown the 30-days mortality, Heart failure, hs-CRP, CK-MB, MELD-XI score at admission were independent predictors.(Table 3). Among all patients admitted, 36 patients(5.2\%) died during admission to hospitalization, 30 days of hospital following discharge. ROC curve analyses revealed that MELD-XI score $\geq 9.78$ had a sensitivity of $75.0 \%$ and a specificity of $64.5 \%$ (AUC: $0.742,95 \% \mathrm{Cl}$ : $0.650-0.833$, $\mathrm{P}<0.001$ ) (Figure 1b). Kaplan-Meier curves revealed that worse outcomes in MELD-XI>9.78 group for 30days all cause mortality $(\mathrm{P}<0.001)$ (Figure 2$)$. 
Table 2

Logistic regression analyses for development of no-reflow phenomenon.

\begin{tabular}{|lllll|}
\hline & Univariate Regression & \multicolumn{3}{c|}{ Multiple Regression } \\
\hline Variable & OR(95\%Cl) & P & OR(95\%Cl) & P \\
\hline Age(years) & $1.010(0.995-1.025)$ & 0.181 & & \\
\hline Male, n(\%) & $0.795(0.499-1.265)$ & 0.333 & & \\
\hline Smoking, n(\%) & $0.691(0.475-1.006)$ & 0.054 & & \\
\hline Heart failure(Killip II-IV,class),n(\%) & $1.774(1.203-2.614)$ & 0.004 & $1.381(0.903-2.112)$ & 0.137 \\
\hline Hypertension,n(\%) & $1.887(1.272-2.797)$ & 0.002 & $1.432(0.936-2.191)$ & 0.098 \\
\hline Diabetes mellitus,n(\%) & $2.256(1.555-3.272)$ & $<0.001$ & $2.173(1.463-3.227)$ & $<0.001$ \\
\hline Stroke or TIA,n(\%) & $1.067(0.583-1.954)$ & 0.833 & & \\
\hline Family history,n(\%) & $1.023(0.407-2.571)$ & 0.962 & & \\
\hline Peripheral vascular history,n(\%) & $5.168(2.294-11.644)$ & $<0.001$ & $3.448(1.412-8.421)$ & 0.007 \\
\hline Hyperlipidaemia,n(\%) & $0.739(0.512-1.068)$ & 0.107 & & \\
\hline SBP, mmHg & $1.009(1.001-1.017)$ & 0.031 & $1.007(0.999-1.016)$ & 0.100 \\
\hline LVEF(\%) & $0.990(0.972-1.010)$ & 0.327 & & \\
\hline TC (mmol/L) & $1.052(0.889-1.245)$ & 0.556 & & \\
\hline TG (mmol/L) & $0.889(0.758-1.041)$ & 0.144 & & \\
\hline hs-CRP (mg/L) & $1.004(1.000-1.008)$ & 0.052 & & \\
\hline CK-MB(U/L) & $1.000(0.999-1.001)$ & 0.939 & & \\
\hline UA( $\mu$ mol/L) & $1.001(0.999-1.002)$ & 0.464 & & \\
\hline MELD-XI score & $1.276(1.176-1.385)$ & $<0.001$ & $1.247(1.144-1.360)$ & \\
\hline OR: Odds ratio; Cl: Confidence interval. & & & \\
\hline
\end{tabular}


Table 3

Cox regression analyses revealing the predictors of 30-days all-cause mortality in patients with STEMI undergoing PPCl

\begin{tabular}{|lllll|}
\hline \multicolumn{4}{l}{ Univariate Regression } & \multicolumn{3}{l|}{ Multiple Regression } \\
\hline Variable & HR(95\%Cl) & $\mathrm{P}$ & HR(95\%Cl) & $\mathrm{P}$ \\
\hline Age(years) & $1.039(1.010-1.069)$ & 0.009 & $1.008(0.978-1.039)$ & 0.606 \\
\hline Male, $\mathrm{n}(\%)$ & $0.617(0.290-1.311)$ & 0.209 & & \\
\hline Smoking, $\mathrm{n}(\%)$ & $0.731(0.377-1.417)$ & 0.353 & & \\
\hline Heart failure(Killip II-IV,class),n(\%) & $3.866(1.993-7.500)$ & $<0.001$ & $2.270(1.017-5.066)$ & 0.045 \\
\hline Hypertension,n(\%) & $2.936(1.286-6.703)$ & 0.011 & $2.144(0.908-5.063)$ & 0.082 \\
\hline Diabetes mellitus,n(\%) & $0.712(0.344-1.477)$ & 0.362 & & \\
\hline Stroke or TIA,n(\%) & $1.147(0.405-3.242)$ & 0.796 & & \\
\hline Family history,n(\%) & $0.670(0.092-4.894)$ & 0.693 & & \\
\hline Hyperlipidaemia,n(\%) & $1.111(0.569-2.172)$ & 0.758 & & \\
\hline Heart rate (bpm) & $1.010(0.989-1.031)$ & 0.360 & & \\
\hline SBP, mmHg & $0.997(0.983-1.012)$ & 0.732 & & \\
\hline LVEF(\%) & $0.954(0.922-0.987)$ & 0.006 & $0.980(0.946-1.016)$ & 0.274 \\
\hline hs-CRP (mg/L) & $1.010(1.005-1.015)$ & $<0.001$ & $1.005(1.000-1.011)$ & 0.036 \\
\hline CK-MB(U/L) & $1.002(1.001-1.004)$ & 0.001 & $1.002(1.001-1.003)$ & 0.005 \\
\hline UA( $\mu$ mol/L) & $1.002(0.999-1.005)$ & 0.190 & & \\
\hline Multivessel vessel,n(\%) & $2.027(0.924-4.447)$ & 0.078 & & \\
\hline MELD-XI score & $1.208(1.140-1.280)$ & $<0.001$ & $1.155(1.077-1.239)$ & $<0.001$ \\
\hline HR: Hazard Ratio; Cl: Confidence interval. & & & \\
\hline & & & \\
\hline
\end{tabular}

\section{Discussion}

Review the entire study, we finally confirmed that : 1) MELD-XI score evaluated at admission were significantly higher in whom with NRP compared to normal flow group. 2) MELD-XI score is a significant independent predictor of NRP in patients with STEMI undergoing PPCI. 3) MELD-XI score had a significant predictive power for for 30-days all-cause mortality in STEMI patients after PPCI. Until then, the predictive role of MELD-XI score in terms of NRP and 30-days all cause mortality in patients with STEMI after PPCl was the first time to study. 
The NRP were arising from multiple factors, while the exact mechanism is complex and still unclear. The main viewpoint including spasm or obstruction of the microcirculation, distal microvascular embolization, long ischemic duration, platelet aggregation, oxidative stress, ischemic or reperfusion injury[11]. Studies over the years have shown that NRP remained a powerful independent predictor of early and long-term death in STEMI patients. Patients who NRP developed experiencing major adverse clinical events(MACEs) and complications more frequently than those with normal flow[12]. Therefore, necessary assessment of NRP and short-term risks should be carried out among the patients of candidate $\mathrm{PCl}$ to further improve the safety of intervention.

At present, several available biomarkers and easily clinical parameters have been found clear evidence for predicting NRP $[13,14]$. The bilirubin is a kind of main bile pigment in human bile and a natural metabolized product of iron porphyrin compounds. The oxidized low-density lipoprotein(oxLDL)-induced ROS production is a harmful cholesterol and a risk factor for atherosclerosis $[15,16]$. Several studies demonstrate that bilirubin as a potent endogenous antioxidant to suppress the reactive oxygen species (ROS). Thus, the bilirubin could inhibit the oxidation process of low-density lipoprotein and have a important role in preventing the progression of atherosclerosis via its antioxidant activity. Endothelial dysfunction may be caused by reactive oxygen species produced by oxidative stress[17]. Previous studies have illustrated that endothelial cell damage caused by excessive oxidative stress shown a strong association with NRP in STEMI patients $[18,19,20]$. Celik et al. examined the associations between TB level with NRP and in-hospital major adverse cardiac events. Moreover, they found a tight association between higher bilirubin level with a lower TIMI flow grade and NRP in STEMI patients[4]. This suggests that bilirubin is key mediator to inhibit inflammatory processes especially accompanied by oxidative stress.

Creatinine is anhydride form of creatine. It is proposes as a marker of the renal function[21]. The kidneys' functional changes can be monitored via the estimation of serum creatinine. Higher serum creatinine at admission is a risk factors of NRP[22]. It has already been demonstrated that mild to moderate renal impairment in STEMI patients underwent PPCl is independently associated with NRP[23]. The exact mechanisms of renal dysfunction and NRP is including the accumulation of elevated reactive oxygen species, inflammatory process and endothelial damage[24]. Endothelial dysfunction was significantly associated with renal dysfunction and impaired myocardial perfusion in STEMI patients[25]. NRP is in part as a result of inflammation-induced reperfusion injury. Several studies found renal dysfunction is also an inflammatory state, while no-reflow phenomenon is associated with inflammatory activity[2628]. There is a certain correlation between the oxidative stress and NRP, which has been confirmed in previous studys[19]. The generation of oxidative stress also plays a significant role in renal impairment[29]. All these condition probably involved in the development of NRP.

Recently, quite a few studies have also shown that elevated total bilirubin has a good predictive value in the prognosis of acute coronary syndrome (ACS) patients underwent PCI[30, 31]. Previous studies has shown that impaired renal function plays a important role in predicting future MACEs in patients with 
PCI[32]. Vinod, P. et al shown that patients who had elevated serum creatinine at admission accompanied with increased risk of developing MACEs[33].

The MELD-XI is a novel and easily-accessible score. It only requires two parameter (TB, sCr) through noninvasive blood test. As a tool to assess liver and kidney function, MELD-XI not only represents the critical condition of patients with organ failure, but is also closely related to in-hospital mortality.

Recently,another study demonstrated that the MELD-XI shows good predictive capacity for in-hospital and one-year mortality in older patients with STEMI undergoing PCI[34]. However, the authors did not explore their conclusion could be extrapolated to other age strata. Our results showed patients with higher MELD-XI score have a higher rate of short-term all-cause mortality. We also found MELD-XI score, CK-MB and hs-CRP at admission in multivariate Cox regression were independent predictors for short-term prognosis. Renal and hepatic function may have high predictive power for mortality of cardiovascular disease. Patients with the no-reflow phenomenon are the highest risk subgroup of patients who with increased risks of early and congestive heart failure and of death. The NRP was consistently associated associated with poor prognosis and high mortality rate of STEMI patients after PPCl. Accordingly, our study find the MELD-XI score showed high predictive power for coronary NRP and short-term prognosis, using to early risk stratification of STEMI patients candidate PPCI.

\section{Limitations}

Several important limitations must be considered in our study. First, this is a cross-sectional and retrospective single-center study does not allow causal inference, and with a small sample size, so bias could not be completely ruled out. Second, our clinical samples only from one regions of China, further external validity of results need to be measured. Third, only end-stage liver disease or on dialysis were excluded, patients with previously diagnosed mild to moderate liver and kidney disease will be retained.

\section{Conclusion}

This study indicated that MELD-XI score predict NRP and 30-days prognosis accurately in STEMI patients undergoing PPCI. MELD-XI could be applied as a early risk stratification tools of recognizing high-risk patients to improve their clinical outcomes.

\section{Abbreviations}

MELD-XI: The model for end-stage liver disease excluding international normalized ratio; NRP: No-reflow phenomenon; TIMI: Thrombolysis In Myocardial Infarction; STEMI: ST-segment elevation myocardial infarction; ACS: acute coronary syndrome PPCl: Primary percutaneous coronary intervention; ROC: Receiver operator characteristic; MACEs: Major adverse clinical events; AUC: Area under the curve; TC: Total cholesterol; TG: Triglycerides; LDL-C: Low density lipoprotein cholesterol; oxLDL: oxidized low density lipoprotein; HDL-C: High density lipoprotein cholesterol; UA: uric acid; BUN: blood urea nitrogen; sCr: serum creatine; TB: Total bilirubin; Nt-proBNP: N-terminal pro-brain natriuretic peptide; CK-MB: 
Creatine Kinase-MB; hs-CRP: high sensitivity C-reactive protein; LVEF: Left ventricular ejection fraction; SBP: Systolic blood pressure; DBP: Diastolic blood pressure; OR: Odds ratio; HR: Hazard Ratio; Cl: Confidence interval; LMCA: Left main coronary artery.

\section{Declarations}

Conflicts of interest

All authors report no potential conflicts of interest with respect to the research.

\section{Acknowledgments}

None.

\section{Author contributions}

ZZW and CLL contributed to design the conception of the study. ZXT, ZL and LZR contributed to the acquisition of data for the work. ZXT and LZR analysed the data and drafted the manuscript. ZZW and CLL put forward on strict and valuable suggestions for the revision of the original manuscript. All gave final approval and agree to be accountable for all aspects of work ensuring integrity and accuracy. All authors read and approved the final manuscript.

\section{Funding}

None.

\section{Availability of data and materials}

The database used and/or analyzed for this study available from the corresponding author on reasonable request (Xin-Tao Zhang; Email: 470480938@qq.com).

\section{Ethics approval and consent to participate}

The study protocol was approved by the Ethics Committee of the Union Hospital affiliated to Fujian Medical University, and the need for informed consent was waived given the retrospective nature of the analysis.

\section{Consent for publication}

Not Applicable.

\section{Competing interests}

The authors declare that they have no competing interests.

\section{Author details}


${ }^{1}$ Department of Cardiology, Union Hospital, Fujian Medical University, 29 Xin-Quan Road, Fuzhou 350001, Fujian, P. R. China

${ }^{2}$ Fujian Institute of Coronary Artery Disease, 350001 Fuzhou, Fujian, China

\section{References}

1. Levine GN, Bates ER, Blankenship JC, Bailey SR, Bittl JA, Cercek B, et al. 2015 ACC/AHA/SCAI focused update on primary percutaneous coronary intervention for patients with ST-elevation myocardial infarction: an update of the 2011 ACCF/AHA/SCAI guideline for percutaneous coronary intervention and the 2013 ACCF/AHA guideline for the management of ST elevation myocardial infarction. J Am Coll Cardiol. 2016;67:1235-50.

2. Abe S, Yoshihisa A, Mai T, Shimizu, T, Nakamura, Y, Yamauchi, H, et al. Liver Dysfunction Assessed by Model for End-Stage Liver Disease Excluding INR (MELD-XI) Scoring System Predicts Adverse Prognosis in Heart Failure. Plos One, 2014, 9(6):e100618.

3. Shafik, Khoury, Gilad, Margolis, Zach, Rozenbaum, Keren-Lee, et al. Acute renal impairment in older adults treated with percutaneous coronary intervention for ST-segment elevation myocardial infarction. Coronary Artery Disease, 2019.

4. Celik T, Kaya M G, Akpek M, et al. Does Serum Bilirubin level on admission predict TIMI flow grade and in-hospital MACE in patients with STEMI undergoing primary PCl. Angiology, 2013, 65(3).

5. Rezkalla SH, Stankowski RV, et al. Management of no-reflow phenomenon in the catheterization laboratory. J Am Coll Cardiol.2017;10:215-23.

6. Soeda T, Higuma T, Abe N, et al. Morphological predictors for no reflow phenomenon after primary percutaneous coronary intervention in patients with ST-segment elevation myocardial infarction caused by plaque rupture. Eur Heart J Cardiovasc Imaging.2016;18:103-10.

7. Iwakura K, Ito H, Takiuchi S, Taniyama Y, Nakatsuchi Y, Negoro S, et al. Alternation in the coronary blood flow velocity pattern in patients with no reflow and reperfused acute myocardial infarction. Circulation 1996; 94:1269-75

8. Thygesen K, Alpert JS, Jaffe AS, et al. Third universal definition of myocardial infarction. Circulation 2012;126:2020-35.

9. Rezkalla SH, Kloner RA. Coronary no-reflow phenomenon: from the experimental laboratory to the cardiac catheterization laboratory. Catheter Cardiovasc Interv. 2008;72:950-957

10. He PC, Wei XB, Luo SN, Chen XL, Ke ZH, Yu DQ, et al. Risk prediction in infective endocarditis by modifed MELD-XI score. Eur J Clin Microbiol Infect Dis. 2018;37:1243-50

11. Bouleti C, Mewton N, Germain S. The no-reflow phenomenon: state of the art. Arch Cardiovasc Dis. 2015;108:661-74.

12. Kaur G, Baghdasaryan P, Natarajan B, et al. Pathophysiology, Diagnosis, and Management of Coronary No-Reflow Phenomenon. International Journal of Angiology, 2021, 30(4). 
13. Zhao, Y, Yang, J, Ji, Y, Wang, S, Wang, T, et al. Usefulness of fibrinogen-to-albumin ratio to predict noreflow and short-term prognosis in patients with ST-segment elevation myocardial infarction undergoing primary percutaneous coronary intervention. Heart Vessels, 2019;34:1600-1607

14. D-Dimer Level Predicts Angiographic No-Reflow Phenomenon After Percutaneous Coronary Intervention Within 2-7 Days of Symptom Onset in Patients with ST-Segment Elevation Myocardial Infarction. Journal of Cardiovascular Translational Research, 2021, 14(4):728-734.

15. Mayer M. Association of serum bilirubin concentration with risk of coronary artery disease. Clin Chem. 2000;46(11):1723-1727.

16. Rigato I, Ostrow JD, Tiribelli C. Bilirubin and the risk of common non-hepatic diseases. Trends Mol Med. 2005;11(6):277-283.

17. Zhang W J, Li P X, Guo X H, et al. Role of moesin, Src, and ROS in advanced glycation end productinduced vascular endothelial dysfunction. Microcirculation, 2017, 24(3).

18. Chen Y, Liu C, Zhou P, et al. Coronary Endothelium No-Reflow Injury Is Associated with ROS-Modified Mitochondrial Fission through the JNK-Drp1 Signaling Pathway[J]. Oxidative Medicine and, Cellular Longevity, 2021, 2021:1-11.

19. Gu“r M, Tu“rkog lu C, Tas, kın A, et al. Paraoxonase-1 activity and oxidative stress in patients with anterior ST elevation myocardial infarction undergoing primary percutaneous coronary intervention with and without no-reflow. Atherosclerosis. 2014;234(2):415-420.

20. Niccoli G, Celestini A, Calvieri C. Patients with microvascular obstruction after primary percutaneous coronary intervention show a gp91phox (NOX2) mediated persistent oxidative stress after reperfusion. Eur Heart J Acute Cardiovasc Care. 2013;2:379-88

21. Bagheri B, Radmard N, Faghani-Makrani A, et al. Serum Creatinine and Occurrence and Severity of Coronary Artery Disease[J]. Medical Archives, 2019, 73(3):154-156.

22. Ahmadreza K, Anbukarasi M. Advances in Coronary No-Reflow Phenomenon-a Contemporary Review. [J]. Current Atherosclerosis Reports, 2018, 20(9):44.

23. Kurtul A, Murat S N, Yarlioglues M, et al. Mild to Moderate Renal Impairment Is Associated With NoReflow Phenomenon After Primary Percutaneous Coronary Intervention in Acute Myocardial Infarction.[J]. Angiology, 2015, 66(7):644-651.

24. Rezkalla S H, Kloner R A. No-Reflow Phenomenon[J]. Circulation, 2002, 105(5):656-662.

25. Stam F, van Guldener C, Becker A, et al. Endothelial dysfunction contributes to renal functionassociated cardiovascular mortality in a population with mild renal insufficiency: the Hoorn study.J Am Soc Nephrol. 2006;17(2):537-545.

26. Filiopoulos $V$, Vlassopoulos $D$. Inflammatory syndrome in chronic kidney disease: pathogenesis and influence on outcomes.Inflamm Allergy Drug Targets. 2009;8(5):369-382.

27. Celik T, lyisoy A, Yuksel UC, Jata B, Ozkan M. The impact of admission C-reactive protein levels on the development of noreflow phenomenon after primary $\mathrm{PCl}$ in patients with acute myocardial infarction: the role of inflammation. Int J Cardiol. 2009;136(1):86-88. 
28. Soylu K, Yuksel S, Gulel O, et al. The relationship of coronary flow to neutrophil/lymphocyte ratio in patients undergoing primary percutaneous coronary intervention. J Thorac Dis. 2013;5(3):258-264.

29. Cottone S, Lorito MC, Riccobene R, et al. Oxidative stress, inflammation and cardiovascular disease in chronic renal failure.J Nephrol. 2008;21(2):175-179.

30. Tang C, Qian H, Wang D, et al. Prognostic Value of Serum Total Bilirubin after Percutaneous Coronary Intervention in Patients with Acute Coronary Syndrome[J]. BioMed Research International, 2019, 2019:1-6.

31. Gul M, Uyarel $\mathrm{H}$, Ergelen $\mathrm{M}$, et al. Prognostic value of total bilirubin in patients with ST-segment elevation acute myocardial infarction undergoing primary coronary intervention.[J]. Journal of the American College of Cardiology, 2013, 111(2):166-171.

32. Kim JY, Jeong MH, Ahn YK, Moon JH, Chae SC, Hur SH, Hong TJ, et al. Decreased glomerular filtration rate is an independent predictor of in-hospital mortality in patients with ST-segment elevation myocardial infarction undergoing primary percutaneous coronary intervention. Korean Circ J. 2011;41(4):184-190

33. Vinod P, Kann T, Polaconda S, et al. The Impact of Admission Serum Creatinine on Major Adverse Clinical Events in ST-Segment Elevation Myocardial Infarction Patients Undergoing Primary Percutaneous Coronary Intervention. Cardiology Research, 2018, 9(2).

34. He S J, Weng J X, Chen H J, et al. The prognostic value of MELD-XI in elderly patients with STsegment elevation myocardial infarction: an observational study. BMC Cardiovascular Disorders, 2021, 21(1)

\section{Figures}



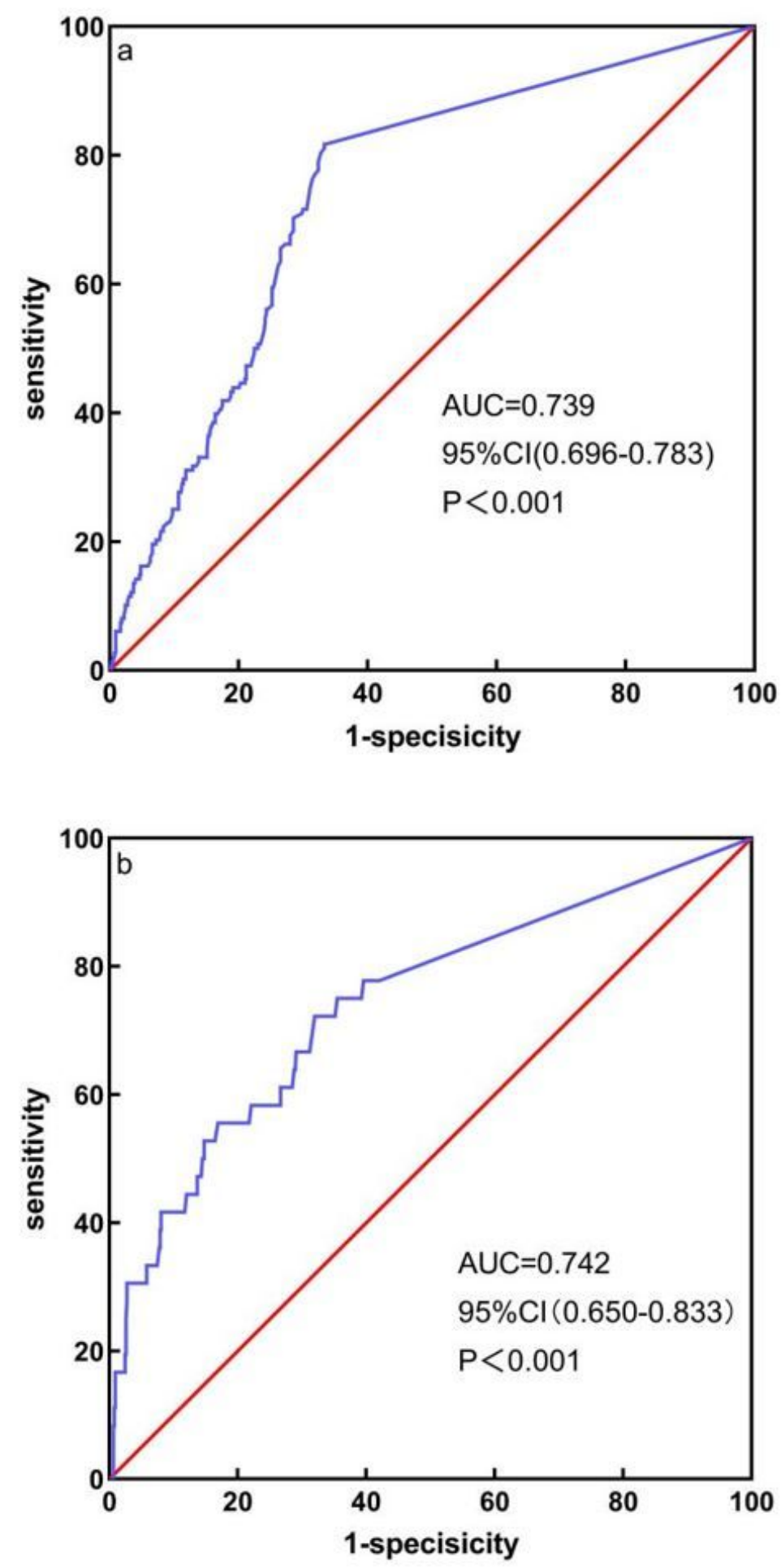

Figure 1

(a). ROC curve of MELD-XI predict no-reflow phenomenon. A sensitivity of $81.90 \%$ and a specificity of $66.67 \%$. (b). ROC curve of MELD-XI predict 30-days all cause mortality. A sensitivity of $75.0 \%$ and a specificity of $64.5 \%$. 


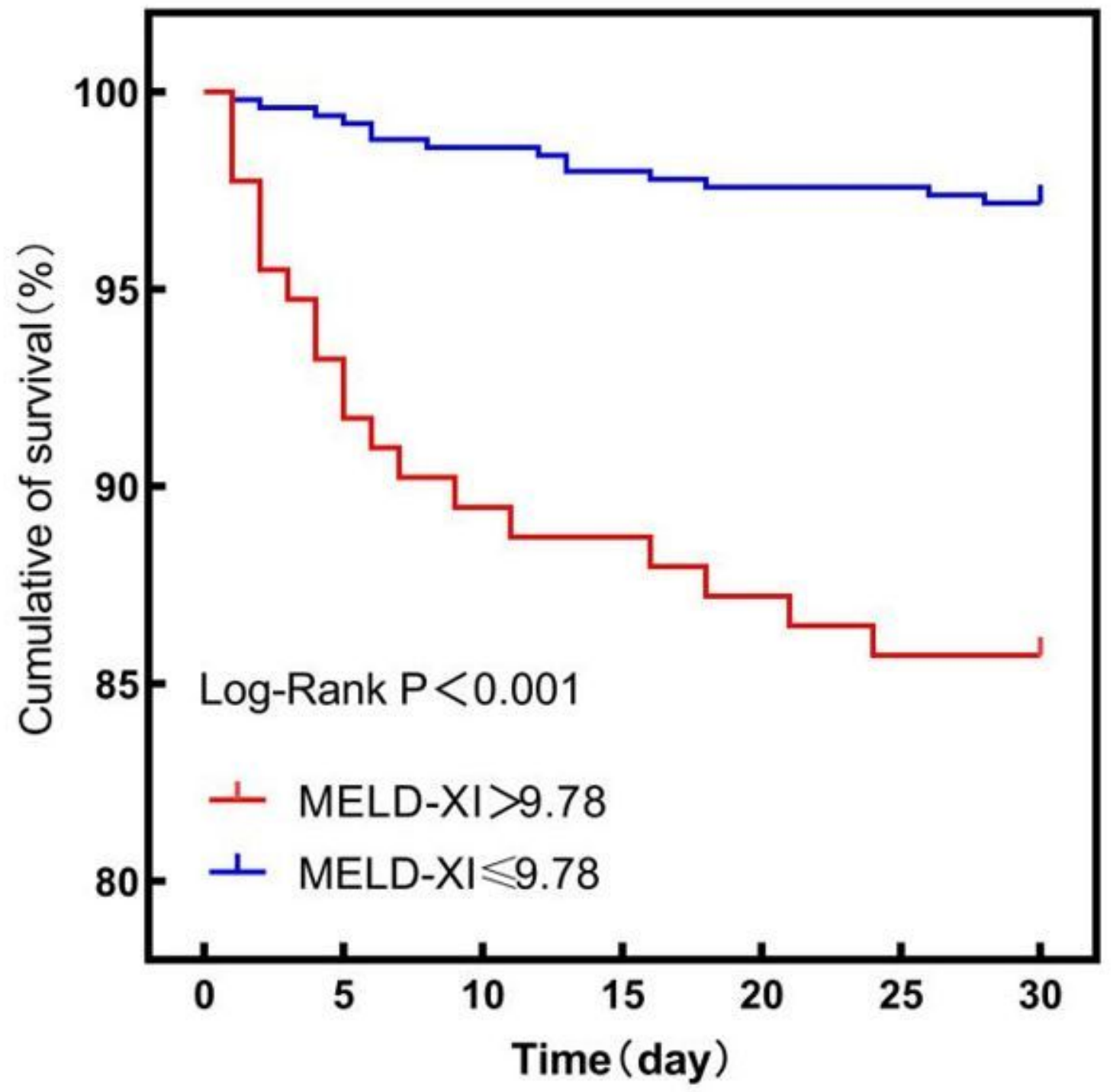

Figure 2

Kaplan-Meier curves of MELD-XI $\leq 9.78$ and $₫ 9.78$ in patients with STEMI undergoing PPCI for 30 -days allcause mortality. 